\title{
Variability in Nucleus Accumbens Activity Mediates Age-Related Suboptimal Financial Risk Taking
}

\author{
Gregory R. Samanez-Larkin, ${ }^{1}$ Camelia M. Kuhnen, ${ }^{2}$ Daniel J. Yoo, ${ }^{1}$ and Brian Knutson ${ }^{1}$ \\ ${ }^{1}$ Department of Psychology, Stanford University, Stanford, California 94305-2130, and ${ }^{2}$ Kellogg School of Management, Northwestern University, Evanston, \\ Illinois 60208-2001
}

As human life expectancy continues to rise, financial decisions of aging investors may have an increasing impact on the global economy. In this study, we examined age differences in financial decisions across the adult life span by combining functional neuroimaging with a dynamic financial investment task. During the task, older adults made more suboptimal choices than younger adults when choosing risky assets. This age-related effect was mediated by a neural measure of temporal variability in nucleus accumbens activity. These findings reveal a novel neural mechanism by which aging may disrupt rational financial choice.

\section{Introduction}

The increases in life expectancy that occurred during the twentieth century will continue to expand the proportion of older adults in the global population (Hayutin, 2007), magnifying the relative economic impact of their financial decisions (Cairncross, 2007). Despite the growing importance of decision competence in old age (Finucane et al., 2002), little research has focused on how aging might influence financial risk taking. Although popular stereotypes suggest that older adults are more risk averse than younger adults, these stereotypes are not well supported by research (Mather, 2006). Instead, research suggests that, in some situations, older adults may simply make more errors when making risky decisions (Denburg et al., 2007; Peters et al., 2007; Mohr et al., 2009). For instance, in the domain of finance, healthy older investors have been shown to continue to invest in risky assets even after suffering losses in the stock market large enough to necessitate postponing retirement (American Association of Retired Persons, 2002).

Age differences in financial decision making could occur for a number of reasons. Extensive research, for instance, has linked age-related deficits in cognitive ability to diminished neural function in the lateral prefrontal and medial temporal cortex (Hedden and Gabrieli, 2004; Cabeza et al., 2005). Beyond cognitive deficits (e.g., related to executive function or memory), aging might also influence value estimation, which might recruit both cortical (e.g., medial prefrontal cortex) and subcortical (e.g., ventral striatum) regions (Knutson and Bossaerts, 2007). In addition to agerelated declines in the structural integrity of the prefrontal cortex

\footnotetext{
Received 0ct. 1, 2009; revised Dec. 1, 2009; accepted Dec. 7, 2009.

This work was supported by National Institute on Aging Grant AG030778, the Financial Industry Regulatory Authority Investor Education Foundation, and Center on Advancing Decision Making in Aging Grant AG024957. G.R.S.-L. is supported by National Institute on Aging Award AG032804. Thanks to Mauricio Delgado, Antonio Rangel, Jesse Rissman, and Brian Wandell for suggestions and Stephanie M. Greer for assistance with data analysis.

Correspondence should be addressed to Gregory R. Samanez-Larkin, Department of Psychology, Building 420, Jordan Hall, Stanford University, Stanford, CA 94305-2130. E-mail: glarkin@stanford.edu.

D. J. Yoo's present address: Department of Psychology, Emory University, Atlanta, GA 30322.

DOI:10.1523/JNEUROSCI.4902-09.2010

Copyright $\odot 2010$ the authors $\quad 0270-6474 / 10 / 301426-09 \$ 15.00 / 0$
}

and striatum (Hicks and Birren, 1970; Rubin, 1999; Buckner, 2004; Head et al., 2005; Raz et al., 2005), theoretical accounts propose that aging may compromise dopaminergic modulation of these regions (Li et al., 2001; Braver and Barch, 2002; Bäckman et al., 2006).

Only a few neuroimaging studies have focused on how aging might influence subcortical function in general (SamanezLarkin and Carstensen, 2010) and striatal function in particular (Aizenstein et al., 2006; Samanez-Larkin et al., 2007; Schott et al., 2007; Dahlin et al., 2008; Dreher et al., 2008; Mell et al., 2009). These emerging findings suggest that, although age may not influence neural responses to explicitly signaled reward cues (Samanez-Larkin et al., 2007) and outcomes (Samanez-Larkin et al., 2007; Cox et al., 2008), age may compromise striatal activity during more cognitively demanding reward tasks (Schott et al., 2007; Mell et al., 2009). Currently, however, no studies have explored age differences in financial decisions related to investments with functional neuroimaging.

Combining computational theories implicating age-related compromises in dopamine function with neuroimaging evidence for altered reward learning, theorists have proposed that "noisy" value signals may bias risky decisions (Li et al., 2007). We tested this proposition by examining whether measures of variance in frontostriatal function might relate to age-related biases in financial risk taking. Specifically, in the context of a financial investment task, we examined whether age might compromise behavioral performance as well as variability in medial prefrontal cortex (MPFC) and nucleus accumbens (NAcc) activity.

\section{Materials and Methods}

Subjects. All subjects were recruited by a survey research firm to be ethnically and socioeconomically representative of San Francisco Bay Area residents. Across the age range, subjects were matched on basic demographic variables (socioeconomic status, income, ethnicity). One hundred ten healthy volunteers (mean age, 51.4; range, 19-85 years; 52\% female) completed the study. All subjects played an investment task, but 54 of these subjects (mean age, 51.3; range, 21-85 years; 54\% female) played the task while undergoing functional magnetic resonance imaging (fMRI). A subset of 38 subjects was specifically not recruited for fMRI as a behavioral control group. Fifty-seven of the remaining 72 adults were 
eligible for $\mathrm{AMRI}$ and completed the scan session. Three of these individuals were excluded from fMRI analyses because of a structural abnormality (71-year-old male) or excessive motion (26-year-old male, 74-year-old male).

All subjects first played a practice version of the investment task. Subjects were then shown the cash they could earn by performing the task successfully. Subjects received a fixed compensation of $\$ 20$ per hour, as well as a tenth of their total earnings during the task. They were also informed that it was possible to lose money on the task and that any losses would be deducted from their total earnings.

Experimental task. A slightly modified version of the Behavioral Investment Allocation Strategy (BIAS) task (Kuhnen and Knutson, 2005) was used to elicit a range of investment behaviors from each subject, including both optimal and suboptimal financial choices. Each subject completed 10 blocks of 10 trials each for a total of 100 trials. During each trial, subjects first saw two stocks and a bond (anticipation, $2 \mathrm{~s}$ ), selected one of these assets when prompted with the word "Choose," and then viewed their highlighted choice on the screen (choice, $4 \mathrm{~s}$ ). After a brief delay (wait, $2 \mathrm{~s}$ ), their earnings for that trial and total earnings were displayed (outcome, $4 \mathrm{~s}$ ) followed by a display of the outcomes of all assets on that trial (market, $4 \mathrm{~s}$ ), and finally a fixation cross (fixation, $2 \mathrm{~s}$ ) (Fig. 1).

At the beginning of each block, the computer randomly assigned one of the two stocks to be the "good" stock, and the other to be the "bad" stock. Subjects were informed that the computer would make these assignments before performing that task but were not informed which stock was assigned to be good and which was assigned to be bad at the beginning of each block. The good stock dominated the bad stock in the sense of first-order stochastic dominance (Huang and Litzenberger, 1988). Specifically, outcomes of the good stock (i.e., $+\$ 10$ with $50 \%$ probability, $+\$ 0$ with $25 \%$ probability, and $-\$ 10$ with $25 \%$ probability) were better than outcomes of the bad stock (i.e., $+\$ 10$ with $25 \%$ probability, $+\$ 0$ with $25 \%$ probability, and $-\$ 10$ with $50 \%$ probability) on average for each trial. The bond paid $\$ 1$ with $100 \%$ probability on each trial. Earnings were drawn independently from these distributions for each trial. After being shown the distributions, all participants were additionally explicitly told that stock choices were riskier than bonds. For instance, an excerpt from the instructions reads: "Once again, the three assets available to choose from are two stocks and a bond. The stocks are risky, because their earnings can be $+\$ 10,-\$ 10$, or $\$ 0$. The bond is riskless, because it always pays $\$ 1$."

Behavioral analysis. In the BIAS task, the optimal strategy of a rational, risk-neutral agent is to pick a stock if he or she expects to receive a dividend that is at least as large as the bond earnings. Since the actual monetary amounts at stake in each trial were small ( $-\$ 1$ to $\$ 1$ ), we used risk neutrality as the baseline model of the rational actor's behavior. A rational actor should also update his or her beliefs about the probability of each stock being optimal according to Bayes' rule. Based on these assumptions, we derived the optimal portfolio selection strategy (for model details, see supplemental material, available at www.jneurosci. org). This optimal model is identical with that applied in previous research using the BIAS task (Kuhnen and Knutson, 2005). To some extent, individual investors approximated the strategy of the rational actor, suggesting that this model provides a reasonable baseline for group comparisons. Like the rational actor, subjects on average showed an initial preference for bonds at the beginning of each block, and then shifted toward preferring the good stock (supplemental Fig. 1, available at www. jneurosci.org as supplemental material).

For each trial, we compared subjects' investment choices with those of the rational actor. Choices that deviated from the rational actor's optimal choices were labeled as suboptimal or "mistakes" and included three types. Risk-seeking mistakes occurred if subjects chose a risky option (i.e., a stock) when the riskless option (i.e., a bond) was the optimal investment. These mistakes tend to occur early within blocks when it is not yet clear which stock is the good stock. Confusion mistakes occurred if subjects chose a risky option (i.e., a stock) when the other risky option (i.e., a stock) was the optimal investment. These mistakes can only be made later within each block when there is enough evidence for the rational actor to distinguish the good from the bad stock. Risk aversion mistakes occurred if subjects chose the riskless option (i.e., the bond)

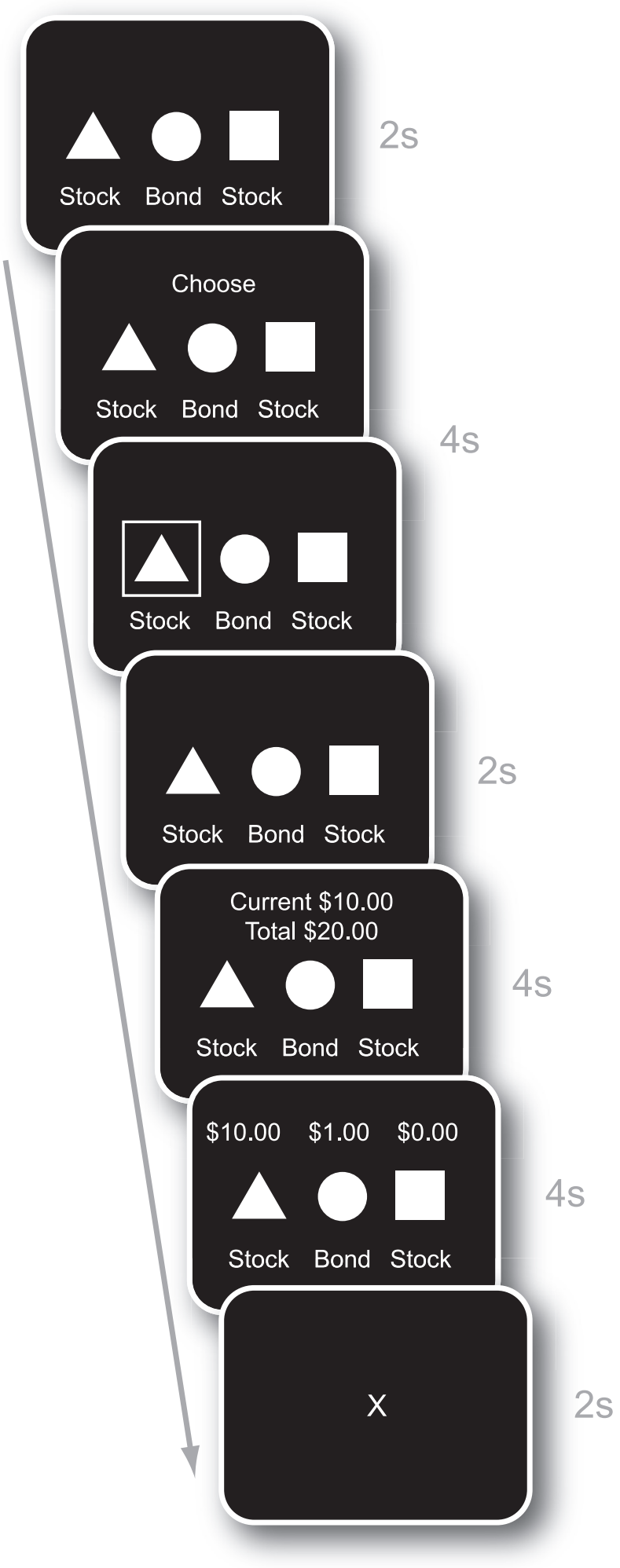

Figure 1. Investment (BIAS) task design. During each trial, subjects first saw two stocks and a bond (anticipation, $2 \mathrm{~s}$ ), selected an asset when prompted with the word "Choose," and then viewed their highlighted choice on the screen (choice, $4 \mathrm{~s}$ ). After a brief delay (wait, $2 \mathrm{~s}$ ), their earnings for that trial and total earnings were displayed (outcome, $4 \mathrm{~s}$ ), followed by a display of the outcomes of all assets on that trial (market, $4 \mathrm{~s}$ ), and finally a fixation cross (fixation, $2 \mathrm{~s}$ ).

when a risky option (i.e., a stock) was the optimal investment. These mistakes also tend to occur relatively later within blocks when the rational actor has enough evidence to distinguish the good from the bad stock. We explored the effect of age on rational choices as well as on each type of mistake. 
fMRI acquisition and analyses. Brain images were acquired with a $1.5 \mathrm{~T}$ GE Healthcare MRI scanner using a standard birdcage quadrature head coil. Twenty-four 4-mm-thick slices (in-plane resolution, $3.75 \times 3.75$ $\mathrm{mm}$; no gap) extended axially from the midpons to the top of the skull, providing adequate spatial resolution of subcortical regions of interest (e.g., midbrain, ventral striatum). Functional scans of the whole brain were acquired every $2 \mathrm{~s}$ [repetition time (TR), $2 \mathrm{~s}$ ] with a T2*-sensitive in/out spiral pulse sequence [echo time (TE), $40 \mathrm{~ms}$; flip, $90^{\circ}$ ] designed to minimize signal dropout at the base of the brain (Glover and Law, 2001). High-resolution structural scans were subsequently acquired using a T1weighted spoiled grass sequence (TR, $100 \mathrm{~ms}$; TE, $7 \mathrm{~ms}$; flip, 90), facilitating subsequent localization and coregistration of functional data.

Preprocessing and whole-brain analyses were conducted using Analysis of Functional Neural Images (AFNI) software (Cox, 1996). For preprocessing, voxel time series were sinc interpolated to correct for nonsimultaneous slice acquisition within each volume, concatenated across runs, corrected for three-dimensional motion, slightly spatially smoothed (full width at half-maximum, $4 \mathrm{~mm}$ ), and high-pass filtered. Statistical maps for individual subjects were coregistered with structural maps, spatially normalized by warping to Talairach space (using manual placement of landmarks in single subjects), and transformed into $Z$ scores. Whole-brain thresholds for statistical significance were set at $Z>3.888, p<0.0001$, with a required cluster size of eight contiguous 2 $\mathrm{mm}$ resampled voxels.

Outcome analyses. Preprocessed time series were submitted to a regression model that included three regressors indexing residual motion and six regressors modeling baseline, linear, and quadratic trends for each of the two runs. Regressors of interest were convolved with a $\gamma$-variate function that modeled a canonical hemodynamic response before inclusion in the regression model (Cohen, 1997). For whole-brain outcome analyses, regressors of interest contrasted stock versus bond choice, as well as gain versus loss outcomes after stock choices. The model also included covariate regressors representing cumulative earnings (defined as current wealth earned during the task, updated at each outcome period) and current trial uncertainty (updated at each market period). For each trial, "uncertainty" referred to the minimum of the objective probabilities (computed using Bayes' rule) of the two individual stocks being dominant.

Temporal variability analyses. In the present study, we used a statistic called the mean squared successive difference (MSSD) (von Neumann et al., 1941) to index the temporal variability (or lability) of fMRI activation. Although this statistic has been used to assess temporal variability of both self-report measures of affect (Woyshville et al., 1999; Jahng et al., 2008) and physiological measures of heart rate variability (Owen and Steptoe, 2003; Berntson et al., 2005), it has not been previously applied to neuroimaging data. MSSD approximates variance but, here, more precisely indexes a lack of temporal specificity of neural activation by computing the variability of the signal from one brain acquisition to the next. For each subject, we calculated the MSSD over the entire preprocessed, detrended, and normalized activation time course averaged and extracted from each of four volumes of interest (VOIs): (1) NAcc, (2) anterior caudate, (3) MPFC, (4) and anterior insula. Given the importance of testing for mediation in evaluating theories of aging (Salthouse, 2006), we then used individual MSSD estimates in a mediation analysis exploring the relationship between age and investment mistakes (Baron and Kenny, 1986). We examine mediation effects both across adult age and within narrow age ranges (Lindenberger and Pötter, 1998; Hofer et al., 2006). Before the mediation analysis, outliers were identified by averaging the MSSD from all four VOIs and excluding individuals $>3$ SDs away from the mean. One subject (70-year-old male) was identified as an outlier and excluded from the temporal variability analyses. Analyses were conducted on the remaining 53 subjects.

VOI definition. VOIs were anatomically specified with 6-mmdiameter spheres in individual subjects based on clusters of activation identified in previous research and based on specific anatomy. The NAcc was defined anatomically (Knutson et al., 2008). The anterior caudate was defined based on the primary cluster of activation from a previous probabilistic learning study that characterized this region as the "actor" in the actor-critic reinforcement learning model (O’Doherty et al., 2004;
Balleine et al., 2007). The other two regions used in the analyses were anatomically defined based on functional effects observed in previous studies in the MPFC (Knutson et al., 2003; Samanez-Larkin et al., 2007) and anterior insula (Samanez-Larkin et al., 2007, 2008). VOI data were used for the temporal variance analyses (described above) and to generate seed time courses for the functional connectivity analyses (described below). For sample VOI placement in four individuals, see supplemental Figure 2 (available at www.jneurosci.org as supplemental material).

Functional connectivity analyses. Using the right NAcc VOI as a seed, functional connectivity analyses examined age and performance differences in frontostriatal connectivity (Draganski et al., 2008) during both anticipation and outcome phases of the task (Rissman et al., 2004). One regression model examined age differences in connectivity and a second regression model examined relationships between individual differences in risk-seeking mistakes and connectivity (controlling for age).

Methodological issues related to age differences. In all analyses, special care was taken to minimize potential confounds associated with age differences (Samanez-Larkin and D'Esposito, 2008). Each individual was screened for dementia and their structural and functional brain imaging data were inspected for abnormalities. Each individual's brain was warped into Talairach space with reference to hand-placed anatomical landmarks. Additionally, all VOIs were anatomically defined on each individual's anatomical images, ensuring that equal amounts of data would be extracted from gray matter in each region for each subject. In this particular study, we did not include a separate hemodynamic response function control (such as hypercapnia or a primary sensory task), but in previous studies examining age differences in striatal regions with similar samples we have included these controls [Samanez-Larkin et al. (2007), their supplemental data], and they did not reveal striking age differences. However, group differences in hemodynamics cannot account for the present effects because similar responses to outcomes were observed across age in the striatum and prefrontal cortex (see Results).

\section{Results}

\section{Behavioral results}

Providing evidence for the ecological validity of behavioral performance in the investment task, a regression analysis revealed a significant relationship between rational choices (i.e., choices that matched the rational actor model) in the BIAS task and the accrual of real-world financial assets, after controlling for debts and age (supplemental Table 2 and supplemental Fig. 3, available at www.jneurosci.org as supplemental material). Subjects who made a higher proportion of rational choices in the investment task also reported accumulating more real-world wealth. Although working memory function [as measured by Letter-Number Sequencing (Wechsler, 1997)] was also correlated with assets $(\beta=0.246 ; t=2.08 ; p<0.05)$, the relationship between rational choices and assets held $(\beta=0.203 ; t=2.20 ; p<0.05)$ after controlling for this index of working memory as well as two other measures of individual differences in cognitive ability [i.e., Digit Span (Wechsler, 1997) and the Trail-Making Test (Delis et al., 2004)].

Rational choices in the task decreased with age. Conversely, investment mistakes in the BIAS task increased with age, as indicated by a significant main effect of age on suboptimal choices $(\beta=0.339 ; t=3.75 ; p<0.0001)$. The effect of age on suboptimal choices remained significant $(\beta=0.265 ; t=2.36$; $p<0.05)$ after controlling for education, numeracy (Lipkus et al., 2001), and performance on Letter-Number Sequencing, Digit Span, and Trail-Making Test. Of these mistakes, however, risk-seeking mistakes $(\beta=0.238 ; t=2.55 ; p<0.05)$ and confusion mistakes $(\beta=$ $0.293 ; t=3.18 ; p<0.05)$ specifically increased with age, whereas risk aversion mistakes did not $(\beta=-0.026 ; t=-0.27 ; p=0.79)$ (Fig. $2 A$ ). When including both linear and quadratic effects of age in the model, the linear effects remained the same but no qua- 
A

Mistakes
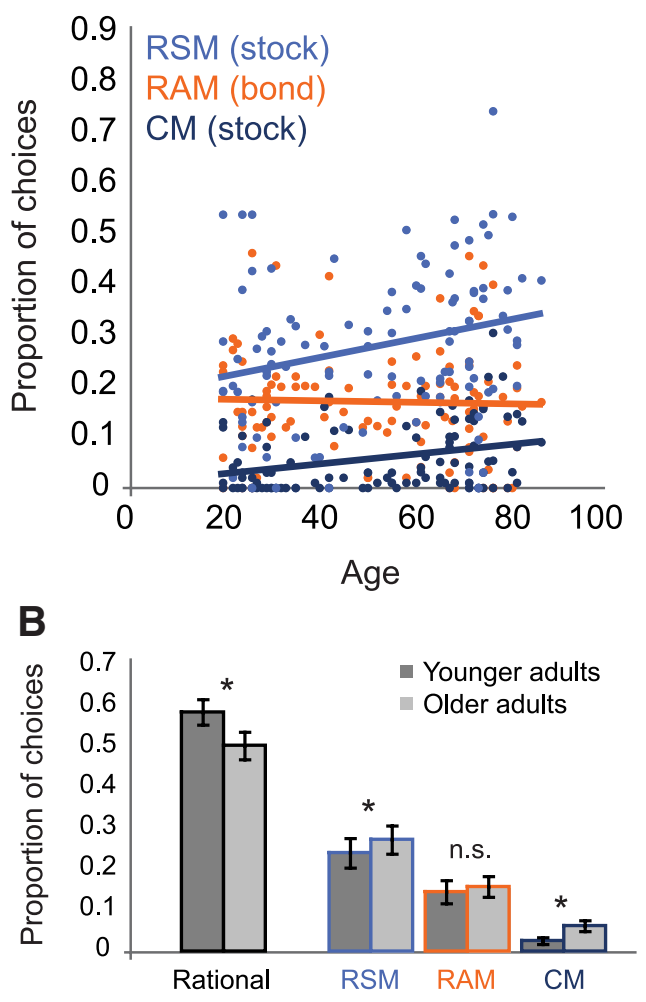

Figure 2. Age-related increase in risky investment mistakes. $\boldsymbol{A}$, Age was associated with increased risk-seeking mistakes (RSM) and confusion mistakes (CM), but not risk aversion mistakes (RAM). $\boldsymbol{B}$, A subset of younger and older subjects who were not recruited for fMRI showed the same behavioral results. * Significant difference between age groups $(p<0.05)$. Error bars represent SEM.

dratic effects were significant (all $p>0.25$ ). Thus, we only report linear effects of age in subsequent analyses.

Although the present community sample was selected to be representative of the demographics of the San Francisco Bay Area, selection may have occurred for the subset of subjects that participated in scanning (e.g., for more risk-seeking individuals). Thus, we ran a follow-up behavioral analysis of age differences in financial risk taking in the subgroup of 19 younger adults (aged 19-30) and 19 older adults (aged 65-81) who did not undergo fMRI. These subjects were only recruited to participate in a behavioral version of the task and no mention of scanning was made to them at any time during recruitment or completion of the study (based on the small sample size and directional predictions, one-tailed tests are reported). In this subset of subjects, the same pattern appeared. Relative to younger adults, older adults made significantly fewer rational choices $\left(t_{(36)}=-2.03 ; p<0.05\right)$, and more risk-seeking mistakes $\left(t_{(36)}=1.47 ; p<0.05\right)$ and confusion mistakes $\left(t_{(36)}=1.64 ; p<0.05\right)$, but did not differ in terms of risk aversion mistakes $\left(t_{(36)}=0.46 ; p=0.33\right)$ (Fig. $2 B$ ).

Although older adults made more mistakes when choosing stocks because of both excessive risk seeking and confusion, riskseeking mistakes occurred much more frequently than confusion mistakes across the entire sample of 110 adults. Specifically, riskseeking mistakes comprised $32 \%$ of choices in the oldest third of subjects aged $67-85$ and $24 \%$ in the youngest third aged 19-35, whereas confusion mistakes comprised $8 \%$ in the oldest third and $3 \%$ in the youngest third. Because of the low incidence of confusion mistakes, the following results focus on explaining age differences in risk-seeking mistakes.
Table 1. Neural activation during monetary outcomes: gain versus loss outcomes across all subjects (controlling for age)

\begin{tabular}{lrrrrr}
\hline Region & R & \multicolumn{1}{l}{ A } & S & Z & Voxels \\
\hline L medial frontal gyrus/cingulate & -10 & 46 & 0 & 4.423 & 16 \\
R middle frontal gyrus & 28 & 38 & -12 & 3.892 & 8 \\
L putamen/nucleus accumbens & -14 & 10 & -8 & 5.678 & 248 \\
R putamen/nucleus accumbens & 14 & 4 & -4 & 4.976 & 192 \\
R posterior cingulate gyrus & 10 & -36 & 34 & 4.405 & 24 \\
R posterior cingulate gyrus & 4 & -40 & 26 & 4.942 & 140 \\
\hline
\end{tabular}

$L$, Left; $R$, right; $A$, anterior; $S$, superior.

$Z>3.888 ; p<0.0001$; eight voxel cluster threshold.

\section{Neuroimaging results}

Neuroimaging analyses sought to identify neural markers that could account for the age differences in investment decision making in the subset of individuals who underwent fMRI. One simple account might posit that age diminishes the strength of neural responses to feedback, which then compromises subsequent reward prediction and choice selection. To examine this possibility, we compared subjects' neural responses to monetary outcomes. Across all subjects, activation in the MFPC, NAcc, anterior caudate, and posterior cingulate (Table 1$)$ increased in response to monetary gain $(+\$ 10)$ versus loss $(-\$ 10)$ outcomes (Fig. 3$)$. An age by outcome interaction revealed significantly greater neural sensitivity to outcomes in older adults in the inferior frontal and temporal gyri (Table 2), but responses to outcomes in the MPFC, NAcc, and anterior caudate did not differ as a function of age. Individual difference analyses evaluated whether sensitivity to outcomes could account for age-related investment mistakes, but none of the regions that showed age-related effects were significantly associated with risk-seeking mistakes (supplemental Fig. 4 and supplemental Table 3, available at www.jneurosci.org as supplemental material). Follow-up VOI analyses confirmed this absence of effects by demonstrating that measures of neural sensitivity to outcomes were not significantly correlated with riskseeking mistakes after controlling for age in the MPFC, NAcc, or anterior caudate (all $p>0.33$ ). Thus, age-related neural responses to feedback could not account for the observed agerelated increases in risk-seeking mistakes.

Although mean anticipatory activity in the NAcc predicted risky (i.e., stock) choices on individual trials (supplemental Table 4) replicating previous findings in younger adults only (Kuhnen and Knutson, 2005), mean activity in the NAcc did not predict risk-seeking mistakes $(p=0.36)$ in this sample that spanned the adult life span.

By an alternative account, temporal variability in NAcc activation might generate mistakes in risky financial decision making (Li et al., 2007). Specifically, if NAcc activation primarily promotes financial risk seeking and becomes noisy (yet not necessarily diminished), this could promote risk-seeking mistakes. We tested this hypothesis by examining whether temporal variability in NAcc activity mediated the relationship between aging and risk-seeking mistakes. As described above, age was associated with risk-seeking mistakes in the subset of subjects who underwent fMRI $(\beta=0.310 ; t=2.23 ; p<0.05)$ (Fig. $4 A)$. Whole-brain analyses revealed that temporal variability (MSSD) increased with age primarily in the NAcc and anterior caudate but not the MPFC. Although the largest cluster had a peak voxel in the thalamus (Table 3), additional peaks within this cluster also appeared in the NAcc and anterior caudate (Fig. 4B). Variability also increased with age in several additional smaller clusters in the midbrain and lateral frontal and parietal cortices (Table 3). 
Follow-up mediation analyses were conducted with temporal variability estimates drawn from each VOI. Age was associated with increased temporal variability in the right NAcc $(\beta=0.490 ; t=3.13 ; p<$ 0.005). Controlling for age, increased temporal variability in the NAcc was associated with increased risk-seeking mistakes $(\beta=0.260 ; t=2.47 ; p<0.05)$. After simultaneous entry of age and NAcc temporal variability into the regression, age no longer significantly predicted riskseeking mistakes $(\beta=0.182 ; t=1.18 ; p=$ 0.12 ), consistent with full mediation of age-related financial risk-seeking mistakes by NAcc temporal variability (Fig. $4 C$ ). The relationship between NAcc variability and risk-seeking mistakes remained significant $(\beta=0.268 ; t=2.67$; $p<0.05$ ) after controlling for education, numeracy, and performance on Letter-Number Sequencing, Digit Span, and Trail-Making Test, in addition to age.

A similar, but weaker, effect was observed in the left anterior caudate. Age was also associated with increased temporal variability in the left anterior caudate $(\beta=0.566 ; t=4.48 ; p<$ $0.0001)$. After controlling for age, anterior caudate temporal variability was marginally associated with increased risk-seeking mistakes $(\beta=0.286 ; t=1.91 ; p=0.06)$, and simultaneous entry of age and anterior caudate temporal variability into the regression revealed that age no longer significantly predicted riskseeking mistakes $(\beta=0.148 ; t=0.96 ; p=0.34)$. The relationship between anterior caudate variability and risk-seeking mistakes was similar $(\beta=0.270 ; t=1.77 ; p=0.09)$ after controlling for education, numeracy, and performance on Letter-Number Sequencing, Digit Span, and the Trail-Making Test in addition to age.

When splitting the sample into thirds by age, the strength of the variability effect appeared to increase with age. Specifically, the relationship between NAcc variability and risk-seeking mistakes (controlling for age) was strongest in the oldest third $(N=$ $18)$ of the sample $(\beta=0.449 ; t=2.29 ; p<0.05)$. There was a trend effect for the middle third of the sample $(N=17)(\beta=$ $0.468 ; t=1.77 ; p<0.10)$, and a nonsignificant effect for the youngest third of the sample $(N=18)(\beta=0.062 ; t=0.24 ; p=$ $0.81)$. This weaker effect in the younger adults is likely attributable to this group's relative lack of measurable neural decline and consequent limited temporal variability.

Importantly, these mediation effects could not be attributed to global increases in temporal variability, since substitution of temporal variability from other brain regions into the model (e.g., left or right MPFC or insula) did not reveal significant associations with risk-seeking mistakes, controlling for age (all $p>0.33$ ). Additionally, temporal variability (MSSD) over the task was a better predictor of risk-seeking mistakes than simple variance of the signal at distinct task phases, since substitution of NAcc signal variance did not predict risk-seeking mistakes (controlling for age) during either anticipation $(p=$ $0.24)$ or outcome $(p=0.11)$.

Functional connectivity analyses explored the possibility that age-related declines in frontostriatal connectivity might also contribute to financial risk-seeking mistakes. Although functional connectivity between the rostral anterior cingulate cortex and NAcc decreased with age both during anticipation (supplemental
Table 2. Neural activation during monetary outcomes: effects of age on gain versus loss outcomes

\begin{tabular}{lrrrrr}
\hline Region & \multicolumn{1}{l}{ R } & \multicolumn{1}{l}{ A } & \multicolumn{1}{l}{ S } & Voxels \\
\hline L inferior frontal gyrus & -32 & 16 & -12 & 4.039 & 16 \\
R superior temporal gyrus & 58 & -10 & 4 & -4.585 & 16 \\
L superior temporal gyrus & -62 & -44 & 18 & 3.923 & 8 \\
R middle temporal gyrus & 46 & -66 & 22 & 4.168 & 16 \\
\hline
\end{tabular}

Positive $Z$ scores indicate greater sensitivity to the difference between monetary gains and losses in older adults. $L$, Left; $R$, right; $A$, anterior; $S$, superior.

$Z>3.888 ; p<0.0001$; eight voxel cluster threshold.

Fig. $5 A$ and supplemental Table $5 A$, available at www.jneurosci. org as supplemental material) and outcome periods (supplemental Fig. 5B and supplemental Table 5B, available at www.jneurosci. org as supplemental material), functional connectivity between these regions was not significantly associated with risk-seeking mistakes (controlling for age). Functional connectivity with other regions also did not correlate with risk-seeking mistakes at the initial whole-brain threshold. At a less stringent statistical threshold ( $p<0.005)$, reduced functional connectivity between the insula/inferior frontal gyrus and NAcc was associated with increased risk-seeking mistakes (supplemental Fig. $5 C$ and supplemental Table $5 C$, available at www.jneurosci.org as supplemental material). Since functional connectivity between these regions did not vary with age, however, it could not account for specific age-related increases in financial risk-seeking mistakes.

\section{Discussion}

The present study investigated age differences in behavior and neural activity in a large community sample of healthy adults as they participated in a dynamic investment task [i.e., the BIAS task (Kuhnen and Knutson, 2005)]. The BIAS task allows comparison of subjects' actual investment choices with those of a "rational" risk-neutral actor who maximizes expected value. Although this investment task is an abstract version of financial decision making, it appears to have some ecological validity. Individuals who make more rational choices in the laboratory also report having accrued more assets in the real world. Despite the growing popularity of laboratory-based financial decision-making tasks, to the best of our knowledge, this represents the first validation of an experimental investment task with real-world financial outcomes. Using this investment task, we found that older adults made more risk-seeking mistakes, and these mistakes were medi- 

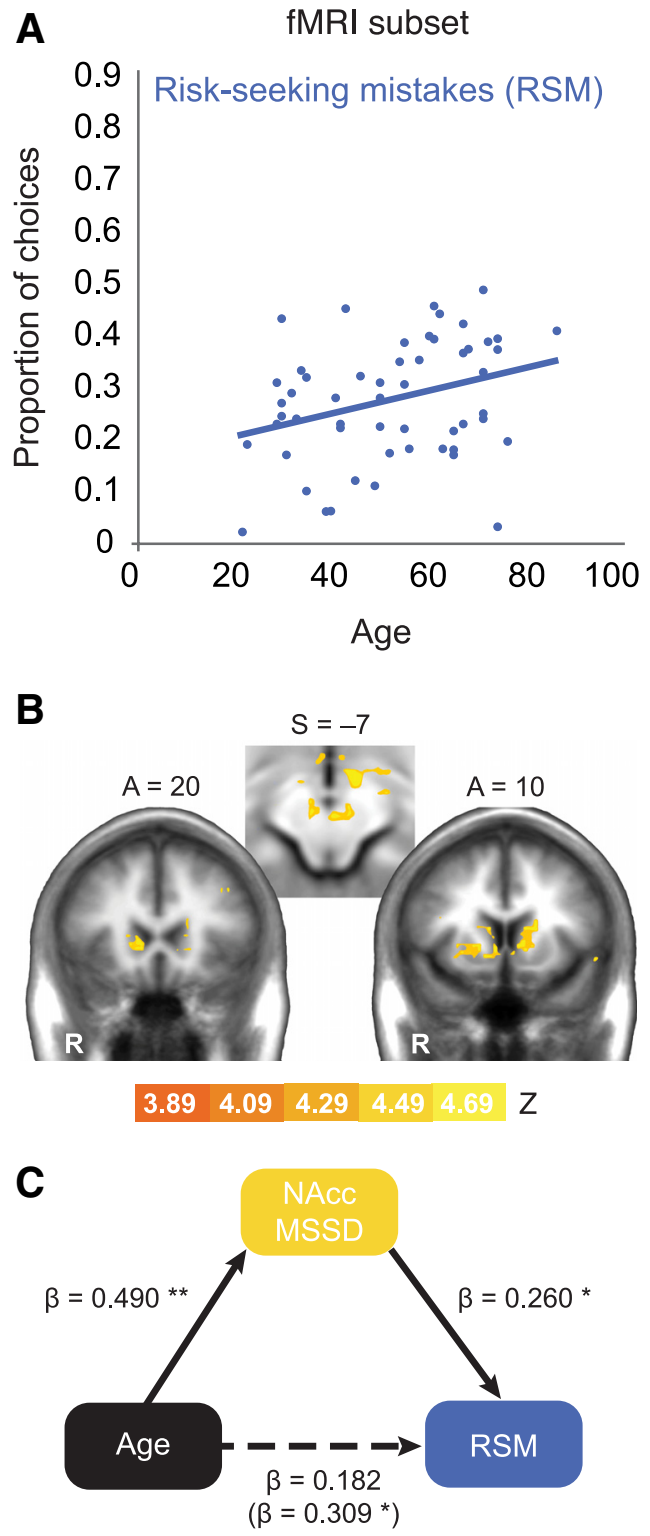

Figure 4. Nucleus accumbens variability mediates age-related risk-seeking mistakes. $A$, Age was associated with increased risk-seeking mistakes (RSM) in the fMRI subject subset. $\boldsymbol{B}$, Temporal variability (MSSD) increased with age throughout the midbrain and striatum with peaks in the substantia nigra, ventral tegmental area $(S=-7)$, anterior caudate $(A=20)$, putamen, medial caudate, and the nucleus accumbens $(A=10)$. Anatomical underlay is an average of all subjects' spatially normalized structural scans. C, Variability in the right NAcc fully mediated the relationship between age and RSM; the relationship between age and RSM became insignificant after adding NAcc variability to the model $(0.309-0.182$; path coefficients are standardized $\beta$ values).

ated by increased temporal variability in the NAcc. The findings thus indicate an age-related subcortical deficit that may promote risky decision-making mistakes.

Although behavioral research does not suggest that aging impairs decision making overall (Mather, 2006), some findings suggest that aging may bias financial decisions (Denburg et al., 2007; Peters et al., 2007; Mohr et al., 2009). In fact, consistent with the present findings, behavioral studies have found that some older adults will persistently choose a risky asset with a negative expected value over a less risky asset with a positive expected value (Denburg et al., 2005), providing additional evidence for agerelated impairments in updating expected value estimates (Mell
Table 3. Age-related increase in temporal variability

\begin{tabular}{lrrrrr}
\hline Region & \multicolumn{1}{l}{ R } & \multicolumn{1}{l}{ A } & Z & Voxels \\
\hline L middle frontal gyrus & -21 & 53 & 16 & 4.267 & 16 \\
R middle frontal gyrus & 31 & 47 & 20 & 4.261 & 13 \\
R anterior cingulate & 7 & 39 & 6 & 4.397 & 12 \\
L middle frontal gyrus & -41 & 23 & 20 & 4.800 & 71 \\
L middle frontal gyrus & -41 & 19 & 36 & 4.817 & 25 \\
L inferior frontal gyrus & -47 & 15 & 22 & 4.360 & 8 \\
L inferior frontal gyrus & -49 & 5 & 22 & 5.066 & 25 \\
L insula & -45 & 1 & 10 & 4.787 & 23 \\
R cingulate gyrus & 9 & 1 & 46 & 4.436 & 8 \\
L amygdala & -27 & -5 & -16 & 4.353 & 12 \\
R precentral gyrus & 53 & -9 & 30 & 4.440 & 14 \\
L precentral gyrus & -49 & -17 & 32 & 4.900 & 71 \\
Ventral tegmental area & -1 & -17 & -6 & 4.853 & 64 \\
R thalamus & 23 & -23 & -2 & 4.893 & 52 \\
L postcentral gyrus & -41 & -25 & 40 & 4.393 & 11 \\
R inferior parietal lobule & 41 & -29 & 44 & 4.536 & 18 \\
L paracentral lobule & -5 & -29 & 52 & 4.315 & 8 \\
R thalamus (extends to NAcc/caudate) & 21 & -31 & 10 & 5.901 & 4522 \\
R inferior parietal lobule & 43 & -49 & 42 & 4.656 & 13 \\
\hline
\end{tabular}

$L$, Left; $R$, right; $A$, anterior; $S$, superior.

$Z>3.888 ; p<0.0001$; eight voxel cluster threshold.

et al., 2005). The present findings cannot be accounted for by differences in investment experience (see supplemental material, available at www.jneurosci.org) and run contrary to popular stereotypes of increasing risk aversion with age. Although adults who undergo brain scans might be more risk seeking than adults in general, additional subjects who completed a behavioral version of the investment task without scanning showed a similar increase in risk-seeking mistakes with age (Fig. $2 \mathrm{~B}$ ). The observed association between age and risk-seeking mistakes also replicated in a separate sample of subjects who were not recruited for brain imaging (Samanez-Larkin et al., 2010).

These findings imply a general decline in the dynamic representation of value (Knutson et al., 2005) with age. This decline may impair older adults' ability to use probabilistic feedback over time to build, alter, and implement optimal value predictions about uncertain future events (Fera et al., 2005). The neuroimaging findings extended those of previous research (SamanezLarkin et al., 2007; Schott et al., 2007; Cox et al., 2008; Mell et al., 2009) by demonstrating that, although age did not disrupt the representation of specific outcomes (i.e., $-\$ 10,+\$ 10$ ) in mesolimbic regions, older adults did not appear to use this feedback as effectively over time to make optimal decisions (Mell et al., 2009).

Novel analyses suggested that increased temporal variability in NAcc activation fully mediated the age-related increase in riskseeking mistakes. This finding is generally consistent with recent evidence for age-related disruptions in the function of dopamine projections (Braskie et al., 2008; Dreher et al., 2008). The finding also more specifically supports the proposition of one computational theory that aging increases variability in neural function (Welford, 1965; Li et al., 2001), extending that proposition to a context that involves financial risk taking.

Variability in dopamine firing, however, may or may not translate into variability in fMRI activity. Alternatively, increased variability in dopamine firing may decrease fMRI activity, particularly when averaged over time. Future studies may more directly test for an association between dopamine firing and neuroimaging signal variability in dopamine target regions with multimodal neuroimaging methods [e.g., positron emission tomography combined with fMRI (Schott et al., 2008)] or by combining neu- 
roimaging methods with pharmacological manipulations of dopamine (Pessiglione et al., 2006). Consistent with translation across levels of analysis, comparative studies suggest a link between phasic dopamine release and the phasic increases in NAcc activity indexed by fMRI (Choi et al., 2006; Knutson and Gibbs, 2007).

Additionally, an fMRI study found increased variability in the prefrontal activation (i.e., residual variance) of individuals who carried a genotype associated with reduced dopaminergic tone [i.e., COMT (catechol-O-methyltransferase) Val carriers] (Winterer et al., 2006). These findings suggest a potential link between age-related changes in dopamine function and variability of activity in specific frontostriatal dopamine targets such as the NAcc.

The novel measure of temporal variability in neural activity used in this study was averaged across the entire scanning session for each subject. The lack of sufficient measurement samples and dynamism of the present design precluded computation of stable mean differences for specific trial phases. Future studies with optimally timed experimental designs might better assess whether this variability is constant across time or related to specific trial phases. Despite these limitations and beyond observing increased temporal variability in the activity of some brain regions innervated by dopamine, we further found support for another key prediction of a computational account (Li et al., 2001). Along with increased temporal variability of neural activity, we also observed reduced discrimination between risky alternatives in older subjects (supplemental Fig. 1, available at www.jneurosci.org as supplemental material).

Age-related variability in NAcc activity may have compromised subjects' ability to accurately predict the value of risky assets, which might have promoted suboptimal choices. In support of this interpretation, more traditional analyses revealed that individuals who make more risk-seeking mistakes show less correlation between NAcc activity and the expected value of risky options (Samanez-Larkin et al., 2010). In a separate behavioral study, risk-seeking mistakes of older adults were reduced by providing visual decision aids indicating the expected value of each risky option (Samanez-Larkin et al., 2010). Together, this evidence suggests that older adults may have difficulty using dynamic probabilistic feedback to predict and select the next best financial option over time.

Although this study focused on ventral striatal activity that mediated age-related mistakes in financial decision making, connected frontal regions may also play important roles specifically in facilitating the integration of value signals over time (Kennerley et al., 2006) and more generally in promoting value-based choice (Hampton et al., 2006; Platt and Huettel, 2008). In the present study, functional connectivity analyses revealed that, independent of age, individuals with reduced connectivity between the NAcc and inferior frontal gyrus/insula made more riskseeking mistakes. Because connectivity between these regions did not vary as a function of age in this sample, however, this relative disconnection could not account for the effects of age on financial risk taking. Although connectivity between the NAcc and anterior cingulate declined with age, connectivity between these regions was not associated with risk-seeking mistakes. This absence of significant effects should not, however, rule out a possible role for frontostriatal disconnections in age-related differences in decision making. Future studies combining structural and functional neuroimaging techniques may more comprehensively address the possible functional consequences of age-related structural disconnections.
Although we did observe a negative association between NAcc and insula connectivity and risk-seeking mistakes, insula activity could not account for age-related differences in choice. Previous studies have found associations between insula activation and representation of risk (Preuschoff et al., 2006) as well as risk prediction error (Preuschoff et al., 2008; d'Acremont et al., 2009). Additionally, a previous study using the BIAS task found that insula activation predicted risk avoidance (i.e., risk aversion mistakes) (Kuhnen and Knutson, 2005) but not excessive risk seeking (i.e., risk-seeking mistakes, the focus of the present study). Thus, one might not necessarily expect to find a relationship between insula activity and risk-seeking mistakes. However, it is also important to note that age differences in insular responses to incentives (Samanez-Larkin et al., 2007) may have limited our ability to detect insular effects.

From a psychological standpoint, one additional explanation for the increase in risk-seeking mistakes in the present sample is that older adults may disproportionately anticipate gains over losses when choosing risky assets. Previous evidence suggests that, although older adults show similar responses to gain and loss outcomes, as well as during anticipation of gains in the absence of learning, they show reduced anticipation of losses, reflected by both self-reported affect and neural activity (Samanez-Larkin et al., 2007). Because of the dynamic and changing nature of the investment task, task-related affect measures were not included, and so this hypothesis could not be directly tested in the present study.

If age-related changes in neural function systematically compromise financial decisions, this might hold significant implications for economic forecasting and policy. Researchers have only begun to empirically explore how individual differences in cognitive capacity might systematically influence financial decisions (Burks et al., 2009; Agarwal et al., 2010; Korniotis and Kumar, 2010). In the present study, older adults made investment errors more frequently than younger adults, possibly because of differences in the representation of expected value. According to this interpretation, variability in NAcc activity diminishes the accuracy of value predictions in older adults, which generates mistakes in financial risk taking. Older adults may find it more difficult to dynamically build value predictions to select the best risky financial option. If so, policy or incentive schemes might consider ways to ameliorate these age-related effects, for instance, by explicitly providing value-based decision aids. Alternatively, policy makers might facilitate more optimal choice among older investors by recommending expert consultation when value computational demands exceed neural capacities (Thaler and Sunstein, 2008).

\section{References}

Agarwal S, Driscoll JC, Gabaix X, Laibson D (2010) The age of reason: financial decisions over the life cycle with implications for regulation. Brookings Papers on Economic Activity, in press.

Aizenstein HJ, Butters MA, Clark KA, Figurski JL, Andrew Stenger V, Nebes RD, Reynolds CF 3rd, Carter CS (2006) Prefrontal and striatal activation in elderly subjects during concurrent implicit and explicit sequence learning. Neurobiol Aging 27:741-751.

American Association of Retired Persons (2002) Impact of stock market decline on 50-70 year old investors. Washington, DC: American Association of Retired Persons.

Bäckman L, Nyberg L, Lindenberger U, Li SC, Farde L (2006) The correlative triad among aging, dopamine, and cognition: current status and future prospects. Neurosci Biobehav Rev 30:791-807.

Balleine BW, Delgado MR, Hikosaka O (2007) The role of the dorsal striatum in reward and decision-making. J Neurosci 27:8161-8165.

Baron RM, Kenny DA (1986) The moderator-mediator variable distinction 
in social psychological research: conceptual, strategic and statistical considerations. J Pers Soc Psychol 51:1173-1182.

Berntson GG, Lozano DL, Chen Y-J (2005) Filter properties of root mean square successive difference (RMSSD) for heart rate. Psychophysiology 42:246-252.

Braskie MN, Wilcox CE, Landau SM, O’Neil JP, Baker SL, Madison CM, Kluth JT, Jagust WJ (2008) Relationship of striatal dopamine synthesis capacity to age and cognition. J Neurosci 28:14320-14328.

Braver TS, Barch DM (2002) A theory of cognitive control, aging cognition, and neuromodulation. Neurosci Biobehav Rev 26:809-817.

Buckner RL (2004) Memory and executive function in aging and AD: multiple factors that cause decline and reserve factors that compensate. Neuron 44:195-208.

Burks SV, Carpenter JP, Goette L, Rustichini A (2009) Cognitive skills affect economic preferences, strategic behavior, and job attachment. Proc Natl Acad Sci U S A 106:7745-7750.

Cabeza R, Nyberg L, Park D (2005) Cognitive neuroscience of aging: linking cognitive and cerebral aging. New York: Oxford UP.

Cairncross F (2007) Economics: age, health and wealth. Nature 448: 875-876.

Choi JK, Chen YI, Hamel E, Jenkins BG (2006) Brain hemodynamic changes mediated by dopamine receptors: role of the cerebral microvasculature in dopamine-mediated neurovascular coupling. Neuroimage 30:700-712.

Cohen MS (1997) Parametric analysis of fMRI data using linear systems methods. Neuroimage 6:93-103.

Cox KM, Aizenstein HJ, Fiez JA (2008) Striatal outcome processing in healthy aging. Cogn Affect Behav Neurosci 8:304-317.

Cox RW (1996) AFNI: software for analysis and visualization of functional magnetic resonance neuroimages. Comput Biomed Res 29:162-173.

d'Acremont M, Lu ZL, Li X, Van der Linden M, Bechara A (2009) Neural correlates of risk prediction error during reinforcement learning in humans. Neuroimage 47:1929-1939.

Dahlin E, Neely AS, Larsson A, Bäckman L, Nyberg L (2008) Transfer of learning after updating training mediated by the striatum. Science 320:1510-1512.

Delis DC, Kramer JH, Kaplan E, Holdnack J (2004) Reliability and validity of the Delis-Kaplan Executive Function System: an update. J Int Neuropsychol Soc 10:301-303.

Denburg NL, Tranel D, Bechara A (2005) The ability to decide advantageously declines prematurely in some normal older persons. Neuropsychologia 43:1099-1106.

Denburg NL, Cole CA, Hernandez M, Yamada TH, Tranel D, Bechara A, Wallace RB (2007) The orbitofrontal cortex, real-world decision making, and normal aging. Ann N Y Acad Sci 1121:480-498.

Draganski B, Kherif F, Klöppel S, Cook PA, Alexander DC, Parker GJ, Deichmann R, Ashburner J, Frackowiak RS (2008) Evidence for segregated and integrative connectivity patterns in the human basal ganglia. J Neurosci 28:7143-7152.

Dreher JC, Meyer-Lindenberg A, Kohn P, Berman KF (2008) Age-related changes in midbrain dopaminergic regulation of the human reward system. Proc Natl Acad Sci U S A 105:15106-15111.

Fera F, Weickert TW, Goldberg TE, Tessitore A, Hariri A, Das S, Lee S, Zoltick B, Meeter M, Myers CE, Gluck MA, Weinberger DR, Mattay VS (2005) Neural mechanisms underlying probabilistic category learning in normal aging. J Neurosci 25:11340-11348.

Finucane ML, Slovic P, Hibbard JH, Peters E, Mertz CK, MacGregor DG (2002) Aging and decision-making competence: an analysis of comprehension and consistency skills in older versus younger adults considering health-plan options. J Behav Decis Mak 15:141-164.

Glover GH, Law CS (2001) Spiral-in/out BOLD fMRI for increased SNR and reduced susceptibility artifacts. Magn Reson Med 46:515-522.

Hampton AN, Bossaerts P, O'Doherty JP (2006) The role of the ventromedial prefrontal cortex in abstract state-based inference during decision making in humans. J Neurosci 26:8360-8367.

Hayutin AM (2007) Graying of the global population. Public Policy and Aging Report 17:12-17.

Head D, Snyder AZ, Girton LE, Morris JC, Buckner RL (2005) Frontalhippocampal double dissociation between normal aging and Alzheimer's disease. Cereb Cortex 15:732-739.

Hedden T, Gabrieli JD (2004) Insights into the ageing mind: a view from cognitive neuroscience. Nat Rev Neurosci 5:87-96.
Hicks LH, Birren JE (1970) Aging, brain damage, and psychomotor slowing. Psychol Bull 74:377-396.

Hofer SM, Flaherty BP, Hoffman L (2006) Cross-sectional analysis of timedependent data: mean-induced association in age-heterogeneous samples and an alternative method based on sequential narrow age-cohort samples. Multivariate Behav Res 41:165-187.

Huang C, Litzenberger RH (1988) Foundations for financial economics. Upper Saddle River, NJ: Prentice Hall.

Jahng S, Wood PK, Trull TJ (2008) Analysis of affective instability in ecological momentary assessment: indices using successive difference and group comparison via multilevel modeling. Psychol Methods 13: $354-375$.

Kennerley SW, Walton ME, Behrens T, Buckley MJ, Rushworth MF (2006) Optimal decision making and the anterior cingulate cortex. Nat Neurosci 9:940-947.

Knutson B, Bossaerts P (2007) Neural antecedents of financial decisions. J Neurosci 27:8174-8177.

Knutson B, Gibbs SEB (2007) Linking nucleus accumbens dopamine and blood oxygenation. Psychopharmacology (Berl) 191:813-822.

Knutson B, Fong GW, Bennett SM, Adams CM, Hommer D (2003) A region of mesial prefrontal cortex tracks monetarily rewarding outcomes: characterization with rapid event-related fMRI. Neuroimage 18:263-272.

Knutson B, Taylor J, Kaufman M, Peterson R, Glover G (2005) Distributed neural representation of expected value. J Neurosci 25:4806-4812.

Knutson B, Delgado MR, Phillips PEM (2008) Representation of subjective value in the striatum. In: Neuroeconomics: decision making and the brain (Glimcher PW, Camerer CF, Fehr E, Poldrack RA, eds), pp 389-406. New York: Academic.

Korniotis GM, Kumar A (2010) Do older investors make better investment decisions? Rev Econ Stat, in press.

Kuhnen CM, Knutson B (2005) The neural basis of financial risk taking. Neuron 47:763-770.

Li SC, Lindenberger U, Sikström S (2001) Aging cognition: from neuromodulation to representation. Trends Cogn Sci 5:479-486.

Li SC, Biele G, Mohr PNC, Heekeren HR (2007) Aging and neuroeconomics: insights from research on neuromodulation of reward-based decision making. Analyse and Kritik 29:97-111.

Lindenberger U, Pötter U (1998) The complex nature of unique and shared effects in hierarchical linear regression: implications for developmental psychology. Psychol Methods 3:218-230.

Lipkus IM, Samsa G, Rimer BK (2001) General performance on a numeracy scale among highly educated samples. Med Decis Making 21:37-44.

Mather M (2006) A review of decision making processes: weighing the risks and benefits of aging. In: When I'm 64 (Carstensen LL, Hartel CR, eds), pp 145-173. Washington, DC: The National Academies.

Mell T, Heekeren HR, Marschner A, Wartenburger I, Villringer A, Reischies FM (2005) Effect of aging on stimulus-reward association learning. Neuropsychologia 43:554-563.

Mell T, Wartenburger I, Marschner A, Villringer A, Reischies FM, Heekeren HR (2009) Altered function of ventral striatum during reward-based decision making in old age. Front Hum Neurosci 3:34.

Mohr PN, Li SC, Heekeren HR (2009) Neuroeconomics and aging: neuromodulation of economic decision making in old age. Neurosci Biobehav Rev. Advance online publication. Retrieved January 14, 2010. doi: 10.1016/j.neubiorev.2009.05.010.

O’Doherty J, Dayan P, Schultz J, Deichmann R, Friston K, Dolan RJ (2004) Dissociable roles of ventral and dorsal striatum in instrumental conditioning. Science 304:452-454.

Owen N, Steptoe A (2003) Natural killer cell and proinflammatory cytokine responses to mental stress: associations with heart rate and heart rate variability. Biol Psychol 63:101-115.

Pessiglione M, Seymour B, Flandin G, Dolan RJ, Frith CD (2006) Dopamine-dependent prediction errors underpin reward-seeking behaviour in humans. Nature 442:1042-1045.

Peters E, Hess TM, Västfjäll D, Auman C (2007) Adult age differences in dual information processes: implications for the role of affective and deliberative processes in older adults' decision making. Perspect Psychol Sci 2:1-23.

Platt ML, Huettel SA (2008) Risky business: the neuroeconomics of decision making under uncertainty. Nat Neurosci 11:398-403.

Preuschoff K, Bossaerts P, Quartz SR (2006) Neural differentiation of ex- 
pected reward and risk in human subcortical structures. Neuron 51:381-390.

Preuschoff K, Quartz SR, Bossaerts P (2008) Human insula activation reflects risk prediction errors as well as risk. J Neurosci 28:2745-2752.

Raz N, Lindenberger U, Rodrigue KM, Kennedy KM, Head D, Williamson A, Dahle C, Gerstorf D, Acker JD (2005) Regional brain changes in aging healthy adults: general trends, individual differences and modifiers. Cereb Cortex 15:1676-1689.

Rissman J, Gazzaley A, D'Esposito M (2004) Measuring functional connectivity during distinct stages of a cognitive task. Neuroimage 23:752-763.

Rubin DC (1999) Frontal-striatal circuits in cognitive aging: evidence for caudate involvement. Neuropsychol Dev Cogn B Aging Neuropsychol Cogn 6:241-259.

Salthouse TA (2006) Theoretical issues in the psychology of aging. In: Handbook of the psychology of aging, Ed 6 (Birren JE, Schaie KW, eds), pp 3-13. New York: Academic.

Samanez-Larkin GR, Carstensen LL (2010) Socioemotional functioning and the aging brain. In: The handbook of social neuroscience (Decety J, Cacioppo JT, eds). New York: Oxford UP, in press.

Samanez-Larkin GR, D'Esposito M (2008) Group comparisons: imaging the aging brain. Soc Cogn Affect Neurosci 3:290-297.

Samanez-Larkin GR, Gibbs SEB, Khanna K, Nielsen L, Carstensen LL, Knutson B (2007) Anticipation of monetary gain but not loss in healthy older adults. Nat Neuroscience 10:787-791.

Samanez-Larkin GR, Hollon NG, Carstensen LL, Knutson B (2008) Individual differences in insular sensitivity during loss anticipation predict avoidance learning. Psychol Sci 19:320-323.

Samanez-Larkin GR, Wagner AD, Knutson B (2010) Expected value infor- mation improves financial risk taking across the adult life span. Soc Cogn Affect Neurosci, in press.

Schott BH, Niehaus L, Wittmann BC, Schütze H, Seidenbecher CI, Heinze HJ, Düzel E (2007) Ageing and early-stage Parkinson's disease affect separable neural mechanisms of mesolimbic reward processing. Brain 130:2412-2424.

Schott BH, Minuzzi L, Krebs RM, Elmenhorst D, Lang M, Winz OH, Seidenbecher CI, Coenen HH, Heinze HJ, Zilles K, Düzel E, Bauer A (2008) Mesolimbic functional magnetic resonance imaging activations during reward anticipation correlate with reward-related ventral striatal dopamine release. J Neurosci 28:14311-14319.

Thaler RH, Sunstein CR (2008) Nudge: improving decisions about health, wealth, and happiness. New Haven: Yale UP.

von Neumann J, Kent RH, Bellinson HR, Hart BI (1941) The mean square successive difference. Ann Math Stat 12:153-162.

Wechsler D (1997) Wechsler Adult Intelligence Scale, Ed 3. San Antonio, TX: Psychological Corporation.

Welford AT (1965) Performance, biological mechanisms and age: a theoretical sketch. In: Behavior, aging, and the nervous system (Welford AT, Birren JE, eds), pp 3-20. Springfield, IL: Thomas.

Winterer G, Musso F, Vucurevic G, Stoeter P, Konrad A, Seker B, Gallinat J, Dahmen N, Weinberger DR (2006) COMT genotype predicts BOLD signal and noise characteristics in prefrontal circuits. Neuroimage 32:1722-1732.

Woyshville MJ, Lackamp JM, Eisengart JA, Gilliland JA (1999) On the meaning and measurement of affective instability: clues from chaos theory. Biol Psychiatry 45:261-269. 